\title{
Une application méconnue et pourtant célèbre de l'électrostatique : les travaux de Marie Curie, de la découverte du radium à la métrologie de la radioactivité
}

\author{
Philippe Molinié \\ Supélec, 3 rue Joliot-Curie, F-91192 Gif-sur-Yvette, France \\ Courrier électronique : philippe.molinie@ supelec.fr \\ Soraya Boudia \\ IRIST, Université Louis Pasteur, 7, rue de l'Université, F-67000 Strasbourg, France
}

\section{Résumé}

L'histoire des sciences, notamment enseignée au grand public, est souvent un récit trop linéaire, reconstruit postérieurement, à la lumière des développements ultérieurs en se focalisant essentiellement sur l'histoire d'un cheminement intellectuel. Un facteur essentiel est souvent négligé : celui des conditions matérielles et sociales de production de la science, qui incluent aussi bien la construction de réseaux et la recherche de financements, que l'instrumentation.

Les travaux récents en histoire des sciences sur Marie Curie, montrent ainsi que la découverte du radium et les premières années d'histoire de la radioactivité doivent être remises en perspective. Nous présentons ici un aspect de ces travaux, à savoir que le fait de disposer d'une technique de mesure bien particulière a joué un rôle essentiel dans l'orientation initiale des travaux de Marie Curie sur les rayons, et a ensuite contribué à imprimer une originalité particulière, pendant plusieurs décennies, à son laboratoire. 
Cette technique est fondée sur la mesure précise de la charge électrique produite dans l'air par la matière radioactive, et est issue directement des travaux de Jacques et Pierre Curie, sur la piézoélectricité et les électromètres. Il nous a paru intéressant de la reconstituer, et de la présenter à la Société Française d'Electrostatique

Keywords: electrometers, radioactivity, Marie Curie, piezoelectricity, charge measurements

\section{Un nouveau regard sur l'histoire des sciences}

"Radioactivité : désintégration spontanée du noyau d'un atome (...) découverte par Henri Becquerel, scientifique français, en 1896." Cette phrase figure dans la plupart des dictionnaires et des ouvrages de physique. Mais que signifie-t-elle?

En 1896, Becquerel annonça effectivement à l'Académie des Sciences que les sels d'uranium présentaient une phosphorescence invisible de longue durée, se distinguant de la phosphorescence ordinaire car ne nécessitant pas d'activation par le rayonnement solaire. Cette découverte ne passa pas inaperçue, et amena d'autres scientifiques connus à s'intéresser à la question, notamment Lord Kelvin, à Glasgow. Pourtant, le sujet n'a pas fait figure de révolution dans le monde de la physique, presque chaque année de cette période apportant la découverte de nouveaux "rayons", tels ceux de Röntgen en 1895. Et les rayons qui faisaient l'objet d'une grande controverse parmi les physiciens, étaient surtout les rayons cathodiques, connus depuis plusieurs décennies, autour desquels se confrontaient partisans de l'éther et tenants de l'hypothèse corpusculaire (ce n'est qu'en 1897 que le travail de J.J. Thomson va prouver que ces rayons sont formés de particules chargées négativement, qui sont un constituant élémentaire de la matière).

On a donc étudié les propriétés des rayons uraniques, mais sans y voir quelque chose de radicalement nouveau. Pour les mettre en évidence, deux techniques furent utilisées : la photographie, qui est à l'origine de leur découverte (comme les rayons $\mathrm{X}$, les rayons uraniques impressionnent les plaques photographiques), et l'électroscope. En reproduisant l'expérience réalisée par Röntgen avec les rayons $\mathrm{X}$, Becquerel avait montré que les rayons uraniques déchargent un électroscope chargé. Comme Kelvin, il utilisa également l'électromètre, pour mesurer la loi de décroissance de la charge électrique d'un condensateur chargé traversé par 
les rayons uraniques. Puis, petit à petit, il semble s'être désintéressé des "rayons uraniques". Il publiera sept articles sur le sujet en 1896, deux l'année suivante, et plus aucun en 1898.

La découverte de Becquerel a donc été une découverte parmi d'autres, jusqu'à ce que Marie et Pierre Curie annoncent la découverte du radium, cette substance qui luisait dans l'obscurité et dont on se rendit compte par la suite qu'elle pouvait faire fondre la glace, sans apport apparent d'énergie extérieure. C'est à ce moment-là seulement que l'on a pris conscience des problèmes physiques et philosophiques que posait cette énergie nouvelle qui semblait violer le principe de Carnot. Le mot "radioactivité" lui-même n'avait été inventé par Marie Curie qu'en 1898, quand il s'avéra que cette propriété n'était pas seulement une propriété de l'uranium. Quant à l'hypothèse de la transmutation des éléments, elle ne sera formulée qu'en 1902-1903 par Ernest Rutherford et Frederick Soddy.

Mais comment les Curie en sont-ils venus à s'intéresser à ces rayons relativement «banals » qu'étaient les rayons uraniques ? En 1897, quand Marie Curie décida de préparer une thèse sur la question, elle ne pouvait pas avoir choisi ce sujet parce qu'il annonçait des développements prometteurs. En réalité, l'explication de l'intérêt et de la démarche de Marie Curie réside probablement en grande partie dans le fait qu'elle avait à sa disposition, au moment des premières publications sur les rayons uraniques, des appareils de mesure qui lui ont donné la possibilité de reproduire les mesures publiées. On peut étayer cette hypothèse par un carnet que nous avons retrouvé dans le fonds Curie à la Bibliothèque Nationale [1], dans lequel sont consignées des notes prises par le couple sur la question des rayons uraniques, et où on relève surtout un intérêt pour les expériences réalisées à l'électromètre, notamment celles publiées par Kelvin, Beattie et Smoluchowski [2]. Concernant Becquerel, la seule note à son sujet est la loi de déviation qu'il mesure avec cet appareil (il n’y a rien par exemple sur les méthodes photographiques, ou sur ses expériences à l'électroscope, pourtant plus nombreuses). C'est donc l'utilisation de l'électromètre qui semble être le fil conducteur de l'intérêt des Curie.

\section{L'électromètre et le parcours des frères Curie}

\subsection{L'électromètre à quadrants ; principe et utilisations}

Tout au long du XIXè siècle, la question de la mesure des faibles quantités d'électricité a reçu des réponses techniques de plus en plus élaborées, en même temps que la théorie 
électrostatique, puis électromagnétique, progressait. Une des plus belles réalisations de ce siècle dans ce domaine, fut l'électromètre à quadrants.

Conçu en 1867 par Lord Kelvin, très prolifique dans ce domaine, son principe est le suivant : une légère aiguille métallique en forme de papillon, accrochée à un pendule de torsion, peut tourner à l'intérieur d'un cylindre aplati fendu verticalement en quatre, de manière à composer quatre quadrants, les quadrants opposés étant reliés sur le plan électrique (Fig. 1). Les deux paires de quadrants sont donc en influence totale sur le plan électrostatique vis à vis de l'aiguille, cette influence se partageant suivant la position de l'aiguille entre les deux paires. L'aiguille est positionnée de telle sorte qu'au repos, elle présente une surface égale face à chaque paire de quadrants. L'application d'une différence de potentiel entre les quadrants, va rendre le montage dissymétrique, et l'aiguille va subir un couple électrostatique. Son mouvement sera visualisé grâce à un petit miroir, qui réfléchira un faisceau lumineux sur une règle graduée, suivant la méthode élaborée par Poggendorf.
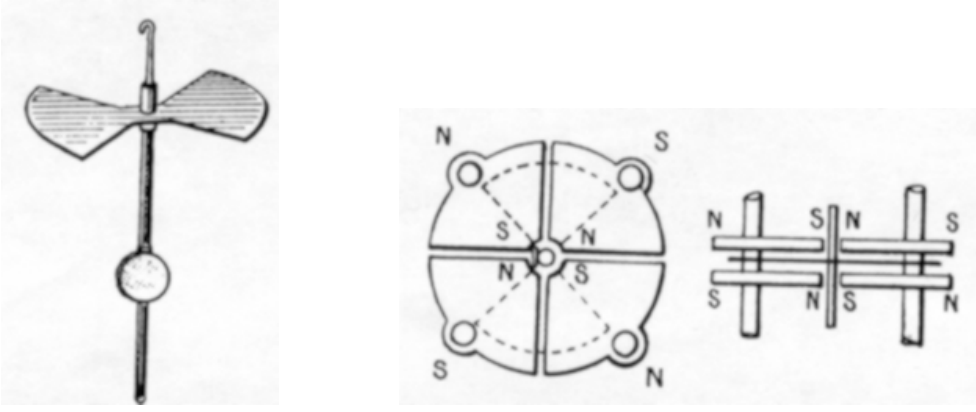

Fig. 1 : Aiguille et quadrants de l'électromètre (d'après Ledeboer [3])

La rotation de l'aiguille est donnée par l'équation :

$$
\theta=\frac{\gamma}{\tau}\left(V_{2}-V_{1}\right)\left(V_{3}-\frac{V_{1}+V_{2}}{2}\right)
$$

$$
\begin{aligned}
& V_{1} \text { et } V_{2} \text { étant les potentiels des quadrants, } V_{3} \text { celui de l'aiguille } \\
& \tau \text { le couple de torsion du fil pour une déviation unité } \\
& \gamma \text { la capacité par unité d'angle de l'aiguille } \\
& \quad \text { (voir par exemple Ledeboer [3] et Mascart [4] ) }
\end{aligned}
$$

Les constantes caractéristiques de l'électromètre sont donc essentiellement le rapport $\gamma / \tau$, qui détermine sa sensibilité, ainsi que sa capacité lorsqu'il est utilisé pour les mesures de charge. Trois utilisations sont possibles, suivant la manière dont est appliquée la tension $\mathrm{V}$ à mesurer : 
1) appliquée à l'aiguille et à un des quadrants, le second quadrant étant relié à la terre, la déviation est donnée par :

$$
\theta=\frac{\gamma}{2 \tau} V^{2}
$$

2) appliquée à l'aiguille, les deux quadrants étant polarisés de manière opposée par deux piles identiques en série $\left(\mathrm{V}_{1}=-\mathrm{V}_{2}=\mathrm{V}_{0}\right)$, on a :

$$
\theta=\frac{2 \gamma}{\tau} V_{0} V
$$

3) appliquée à l'un des quadrants, l'aiguille étant polarisée par une tension constante $V_{0}$, on a :

$$
\theta=\frac{\gamma}{\tau} V\left(V_{0}-\frac{V}{2}\right)
$$

Ce montage (appelé hétérostatique), est utilisé seulement pour des tensions à mesurer nettement inférieures à la polarisation d'aiguille. On a alors :

$$
\theta \approx \frac{\gamma}{\tau} V_{0} V
$$

La première solution, peu sensible et non linéaire, n'est pas retenue pour des mesures de tensions faibles. La deuxième, qui sera notamment utilisée avec l'électromètre Mascart, est la plus « universelle » car elle permet de mesurer linéairement des tensions faibles ou élevées, la relation restant linéaire ; elle sera utilisée notamment pour l'étude de l'électricité atmosphérique. En revanche, elle a l'inconvénient pour les tensions très faibles de nécessiter une égalité rigoureuse entre les deux piles; elle est donc mal adaptée pour une méthode de zéro, pour laquelle seul le montage hétérostatique sera satisfaisant. C'est à ce montage que nous allons nous intéresser.

L'appareil complet a connu différentes versions, dont la plus complexe, et la plus raffinée, semble avoir été la version initiale (voir Fig. 2, et Kelvin [5]). Cet appareil est conçu pour l'utilisation avec le montage hétérostatique. L'aiguille, suspendue par deux fils de cocon isolants, est chargée au moyen d'un fil conducteur baignant dans une cuve remplie d'acide sulfurique. La fonction de cette cuve est d'une part, d'assurer un desséchement efficace de l'atmosphère de l'appareil pour réduire les fuites, et d'autre part, de faire bouteille de Leyde, c'est à dire condensateur, afin de maintenir constante la charge de l'aiguille, l'appareil étant prévu pour être autonome, sans batterie extérieure. L'aiguille est 
chargée par un "rechargeur" et on peut vérifier sa charge au moyen d'une jauge, qui est ellemême un petit électromètre installé sur l'appareil. Sans pile, on peut donc charger l'aiguille à des potentiels assez élevés, et donc avoir une grande sensibilité, en mesurant des tensions relativement élevées par le montage hétérostatique. Cependant, quand les tensions à mesurer sont élevées, la déviation de l'aiguille devient trop grande. Pour répondre à ce problème, il est prévu dans ce cas de mesurer indirectement la tension, par influence, en l'appliquant à une petite plaque d'induction voisine d'un des quadrants.

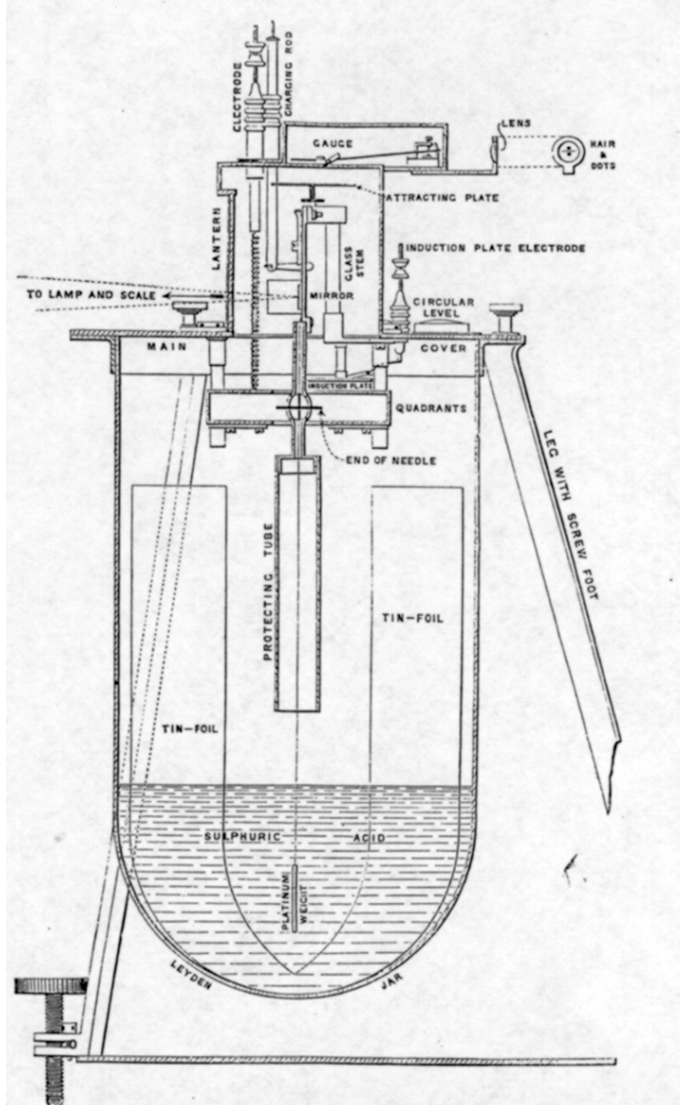

Fig. 2 : Electromètre à quadrants Kelvin (d'après Lefèvre, Dictionnaire d'électricité, 1895)

Il y a eu ensuite différentes versions de cet appareil, simplifiées, qui chacune sera orientée vers un besoin spécifique. Dont celles développés par Pierre Curie, qui seront utilisées essentiellement pour des méthodes de zéro. 


\subsection{Les travaux de Jacques et Pierre Curie}

Jacques Curie, le frère aîné de Pierre, ayant mené au laboratoire de minéralogie de la Faculté des Sciences de Paris des travaux sur l'électricité induite par les cristaux, aboutit à la découverte de la piézoélectricité en 1880. Puis, en 1888, il soutint une thèse sur la conductibilité des isolants où il établit ce que l'on connaît aujourd'hui sous le nom de loi de Curie-Von Schweidler. Quant à Pierre, il avait assisté Jacques presque dès le début, et pris une part déterminante dans la découverte de la piézoélectricité, et cette période lui avait permis de se familiariser avec les mesures de charge au moyen de l'électromètre à quadrants. Dans la décennie suivante, il avait à la fois poursuivi une réflexion théorique sur la symétrie, dont la portée ne sera reconnue que plus tard, effectué ses célèbres travaux sur le magnétisme, et conçu ou amélioré plusieurs instruments de mesure, dont des électromètres. Dans ce foisonnement, nous ne développerons ici que cet aspect, relatif aux instruments de mesure des charges électriques.

\subsection{Le quartz piézoélectrique}

La découverte de la piézoélectricité a mené les frères Curie à construire un générateur de quantités déterminées de charges électrostatiques (voir [6] et [7]). Cet appareil est construit autour d'une fine lame de quartz taillée de manière particulière, dont les deux faces sont métallisées, de telle sorte que l'on puisse collecter la charge électrique apparaissant lors d'une traction sur la lame. Un plateau permet de déposer des poids, et donc de générer des quantités de charges proportionnelles à ces poids, selon la relation :

$$
q=\frac{K l p}{e}
$$

K coefficient caractéristique du quartz, 1 longueur de la lame, e épaisseur.

Ce générateur de charge est donc caractérisé par la constante $K l / e$, et par sa capacité. En effet, la charge produite va se répartir entre la capacité propre de l'appareil et celle du circuit auquel il est connecté, de manière proportionnelle au rapport des capacités. Pour mesurer les courants faibles, on utilise la production calibrée de charges par le quartz en compensation de la source de courant que l'on veut mesurer, la mesure étant effectuée par le maintien de zéro volt sur l'électromètre (voir montage Fig. 5). Cette méthode de zéro permet donc d'utiliser l'électromètre avec sa plus grande sensibilité, tout en minimisant les fuites. Par ailleurs, c'est un instrument qui permet de produire des quantités d'électricité dans un rapport de un à dix mille, puisqu'on peut lui appliquer des tractions variables entre $0,5 \mathrm{~g}$ et $5 \mathrm{~kg}$. 


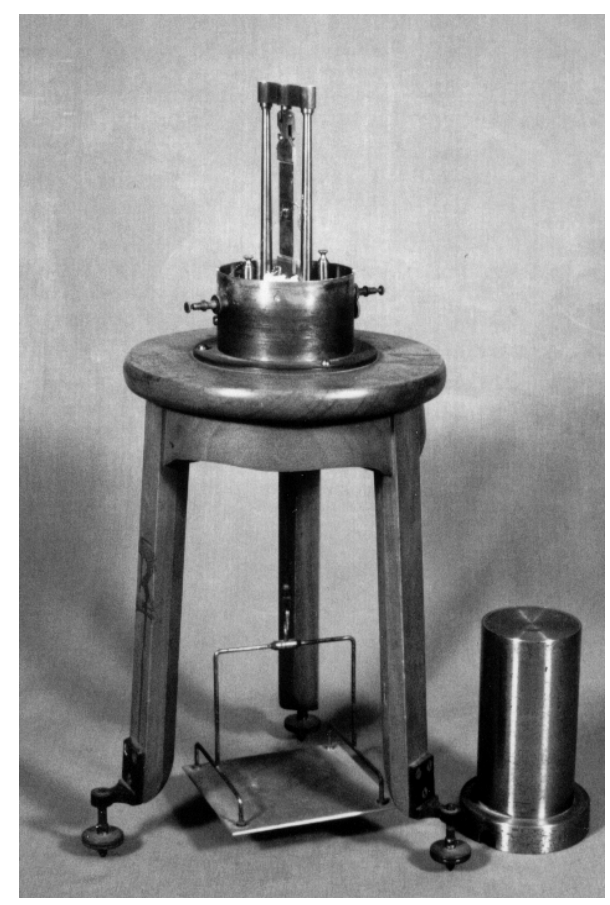

(photo S.B.-B.V.- ESPCI)

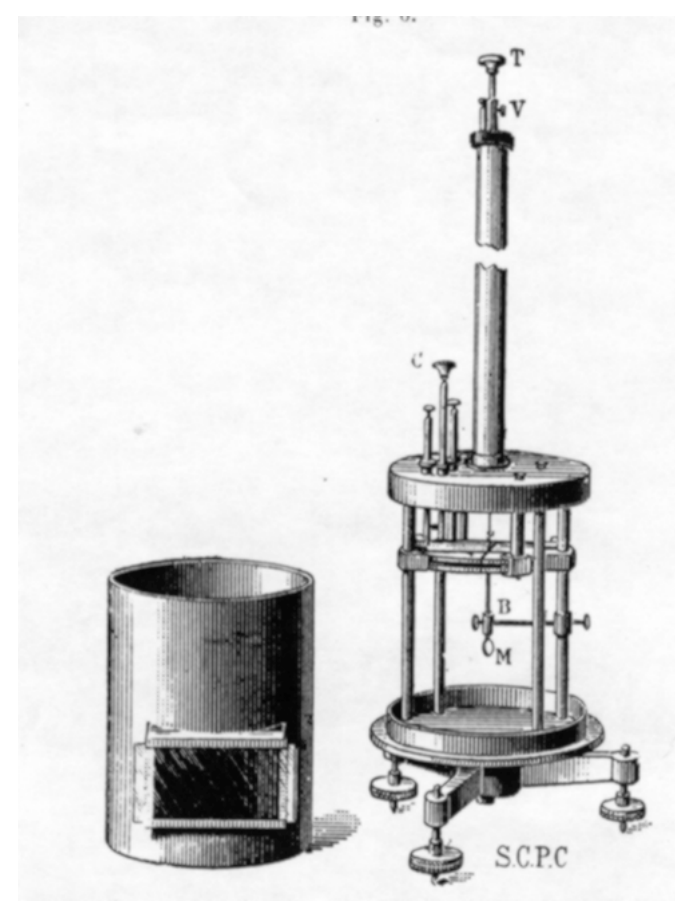

(d'après Ledeboer [3])

Fig.3: Quartz piézoélectrique, et électromètre apériodique Curie

\subsection{Les électromètres Curie}

Pierre Curie a travaillé longuement à l'amélioration des appareils, et notamment des électromètres. Il s'est préoccupé des questions d'amortissement, qui sont d'une importance critique pour ces appareils dont l'aiguille a tendance à osciller longuement avant d'atteindre sa position d'équilibre. Dans le modèle de Kelvin, c'est la rotation d'un petit cylindre solidaire de l'aiguille dans la cuve d'acide qui assure cet amortissement. Pour que le système soit apériodique, l'amortissement doit être égal ou supérieur à $2 \sqrt{\tau \sum m r^{2}},\left(\sum m r^{2}\right.$ étant la somme des moments d'inertie de l'appareillage tournant). Cependant, si l'amortissement est trop important, le retour à l'équilibre peut être extrêmement long et la mesure faussée.

Curie calcula théoriquement le coefficient d'amortissement qui assure le retour à l'équilibre le plus rapide [8], et proposa une version de l'électromètre où l'amortissement est assuré par courants de Foucault dans l'aiguille, les quadrants étant réalisés en acier aimanté. Ce montage se révélant décevant, car l'aiguille avait tendance à se coller aux quadrants, il proposera finalement d'en rester à l'amortissement par l'air sur l'aiguille, accentué autant que possible en réduisant l'épaisseur des quadrants. 
Par ailleurs, il remplacera la suspension en fils de cocon de Kelvin, ou le fil de platine qui était aussi très utilisé, par un fil de quartz, réduisant ainsi le couple de torsion et augmentant considérablement la sensibilité de l'électromètre. Enfin, l'apparition de nouveaux isolants moins sensibles à l'humidité, d'abord l'ébonite, puis l'ambroïde, vont permettre de faire disparaître l'acide sulfurique, et de se contenter de produits désséchants solides. Comme le quartz piézoélectrique, les électromètres apériodiques élaborés par Pierre Curie seront construits, et commercialisés successivement par Bourbouze (1885, modèle avec aimantation), puis par la Société Centrale des Produits Chimiques à partir de 1890 (modèle à amortissement par air). (Sur tous ces aspects, voir Ledeboer [3]).

\section{Des "rayons uraniques" au radium, la reconstitution du parcours expérimental de Marie Curie}

\subsection{La mise en place du système de mesure, retracée d'après les carnets de laboratoire}

Au moment où Marie Sklodowska se maria avec Pierre Curie, en 1895, ce dernier était donc déjà un physicien reconnu, même si il n'avait ni un poste, ni des moyens à la hauteur de son mérite scientifique. Pierre avait participé aux débats sur les rayons cathodiques l'année précédente, en étant plutôt partisan de l'hypothèse corpusculaire. Et il avait un appareillage précis des mesures de courant, ainsi qu'une bonne expérience de la question. Comme on l'a mentionné, l'existence de cet appareillage a eu une importance décisive dans les choix de Marie, même si, paradoxalement, le cheminement «instrumental » des Curie est presque invisible dans les articles qu'ils ont publiés. Pour le reconstituer, nous nous sommes appuyés sur une source de première main : les carnets de laboratoire, qui sont une source parcellaire, mais que nous avons cherché à exploiter de la manière la plus complète possible [9]. Ils couvrent la période de 1897 à 1899, pendant laquelle Marie Curie, avec l'aide de Pierre, mit en place dans des conditions précaires, à l'Ecole municipale de physique et chimie industrielles, un système de mesure précis du rayonnement, étudia de multiples minerais, découvrit l'activité du thorium, et enfin traita la pechblende pour en isoler deux nouveaux éléments bien plus radioactifs que l'uranium, le polonium d'abord, puis le radium [10]. 
Grâce aux carnets, nous pouvons suivre presque quotidiennement leur travail à partir du 16 décembre 1897. L’objectif de Marie était de reproduire les expériences de Kelvin, Beattie et Smoluchowski en vue de quantifier la conductibilité de l'air sous l'effet des rayons uraniques. Dans un premier temps, elle connecte une paire de quadrants d'un électromètre à un des plateaux d'un condensateur, l'autre plateau sur lequel elle dépose les substances à étudier étant chargé par une série de piles. Elle mesure alors la vitesse de déviation de l'électromètre, ce qui lui donne accès à une variation de tension par unité de temps. Connaissant la capacité de l'ensemble condensateur + électromètre, cela doit en principe donner accès au courant traversant le condensateur sous l'influence des rayonnements.

Puis, après plusieurs séries de mesures, le 20 janvier 1898, elle introduisit dans le montage le quartz piézo-électrique. Grâce au dispositif de compensation évoqué plus haut, la mesure de la charge traversant le condensateur est alors effectuée par le dépôt ou le retrait progressif de poids posés sur le plateau accroché à la lame de quartz, de manière à maintenir le spot de l'électromètre le plus précisément possible à zéro. Après quelques difficultés initiales, liées notamment à un défaut d'isolement d'un des appareils, le système sera opérationnel, Marie Curie s'étant familiarisée avec le montage et ayant acquis le "tour de main" nécessaire.

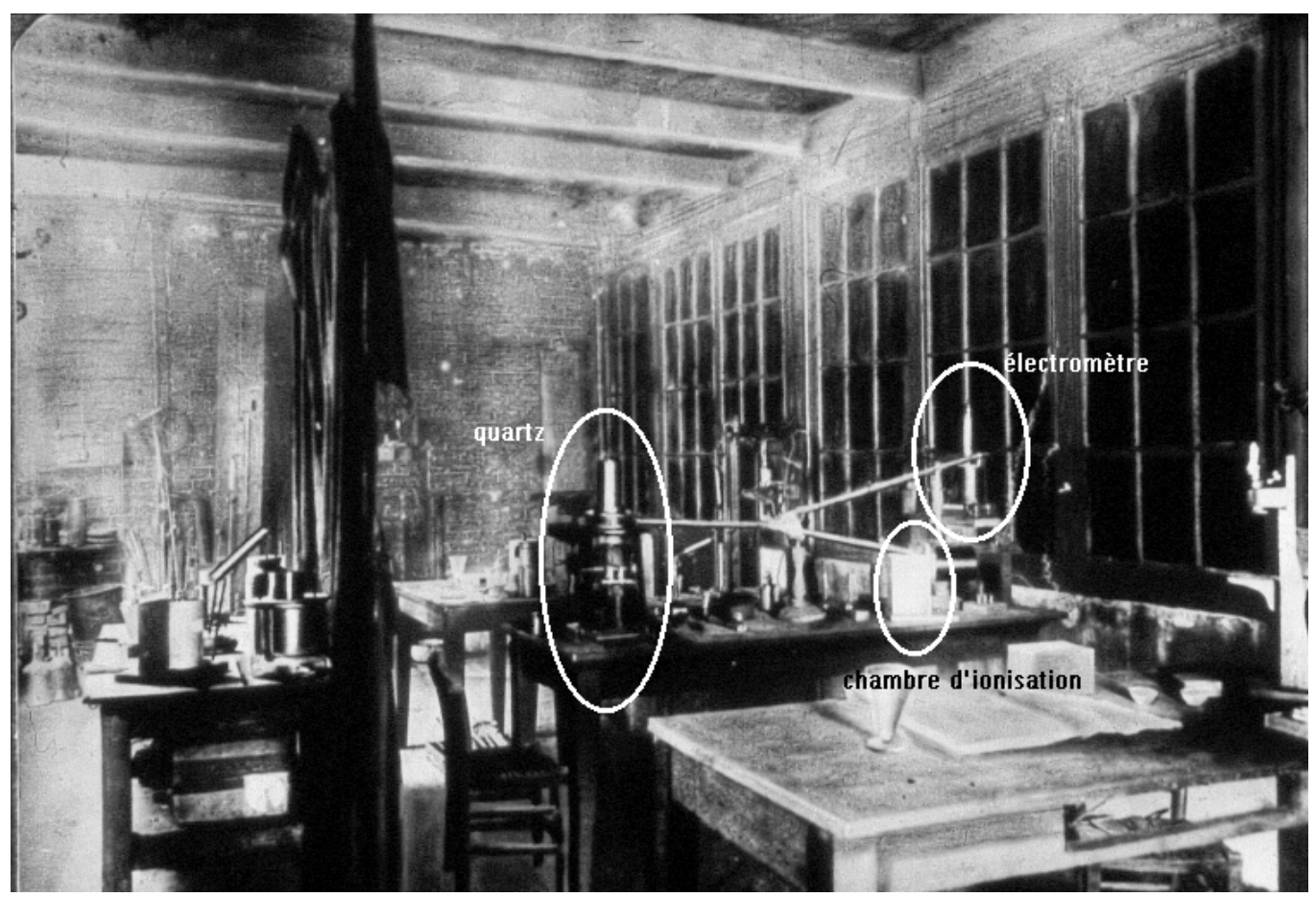

Fig. 4 : Le célèbre hangar de la découverte, avec le banc de mesure (photo 1903 - CACJC) 
Ce qui est notable, c'est que jamais ne sont mentionnés par la suite dans les carnets de quelconques modifications du dispositif quartz - électromètre : un changement de lame de quartz, d'électromètre, ou seulement de fil de suspension de l'aiguille, aurait modifié les coefficients multiplicatifs du système, et serait certainement apparu dans les carnets. En revanche, le condensateur sera changé à plusieurs reprises. Il semble donc que, dès le départ, les Curie disposaient avec le quartz et l'électromètre d'un système assez performant, bien adapté à la mesure précise des rayonnements. On verra cependant plus loin que ce système nécessitait bien des précautions d'emploi, au point qu'il découragera bien des expérimentateurs. On peut donc en conclure que Marie profita largement, non seulement des appareils, mais surtout du savoir-faire accumulé par Pierre et Jacques dans les années précédentes.
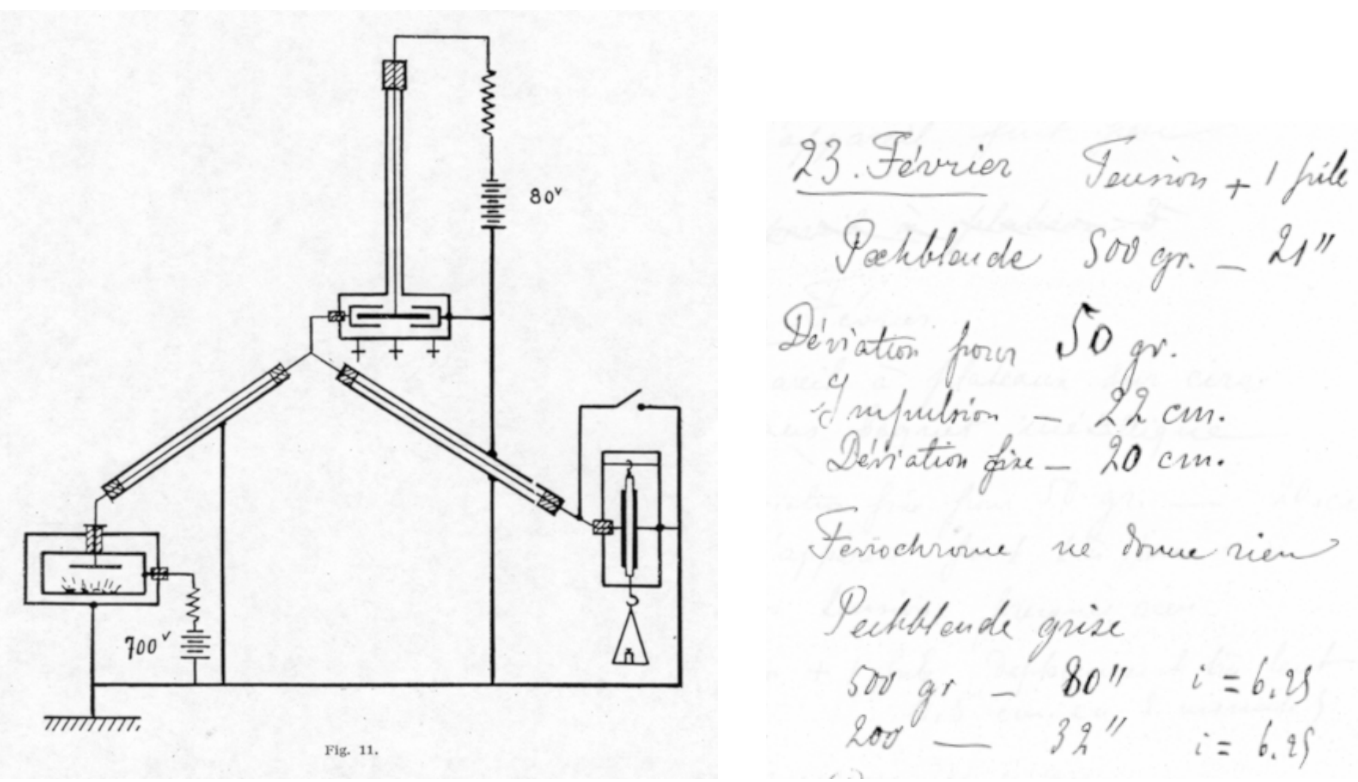

Fig. 5 : Schéma du montage (selon la notice Beaudoin de 1927 [12]) et extrait d'une page du cahier de mesures de Marie Curie [9]

Marie Curie essaya de vérifier si la propriété d'émettre des « rayons uraniques » était propre à l'uranium ou non, ce que d'autres scientifiques avaient entrepris avant elle, mais elle fut la seule à avoir entrepris une recherche systématique, après avoir réuni de très nombreux corps. Et le système expérimental de mesures original Curie permettait à Marie de quantifier précisément l'activité de chaque substance. Grâce aux relations de Pierre, elle put se procurer les matériaux et minerais variés, et put donc procéder à des comparaisons, de telle sorte qu'après deux mois et demi de travail, elle parvint à établir d'une part, qu'un minerai d'uranium, la pechblende, était plus actif que l'uranium lui-même, et d'autre part, qu'un autre minerai, celui du thorium, était également actif. 


\subsection{L'analyse du système de mesure et la tentative de le reconstituer}

\section{La reproduction d'une expérience}

Pour essayer de comprendre plus en détails ce parcours expérimental, nous avons reconstitué, il y a quelques années, le système de mesures utilisé par Marie Curie. La reconstitution d'une expérience présente un intérêt pour comprendre les difficultés qui ont pu se présenter, ce qui a pu guider les choix, aspects qui ne sont pas forcément mentionnés dans le récit de la découverte. Ainsi, la reproduction de l'expérience de Coulomb, qui lui a permis en 1785 de formuler la loi de la force électrostatique grâce à sa balance de torsion, a montré la difficulté extrême à manipuler cet instrument, et que les résultats obtenus devaient au moins autant à l'intuition de Coulomb sur le résultat qu'il devait obtenir (en $1 / \mathrm{r}^{2}$ ) qu'aux mesures elles-mêmes. Concernant Marie Curie, cela posait de nombreuses difficultés, car les carnets, comme on l'a dit, ne contiennent que très peu de détails sur l'électromètre et le quartz utilisés. Les instruments originels ont très probablement disparu ; certaines chambres d'ionisation de l'époque sont conservées à l'ESPCI mais ne sont pas forcément celles qui sont décrites dans les carnets. Par ailleurs, tout l'appareillage utilisé pendant les premières années est contaminé, de telle sorte qu'il serait impossible de s'en servir aujourd'hui. Nous avons donc utilisé des instruments des années 1920, fabriqués par la société Beaudoin, versions légèrement modernisées des modèles des années 1895-1900.

\section{L'électromètre, le quartz et les connexions}

La mise en place du banc de mesure est une opération délicate [12]. L'installation de l'aiguille et de son système de suspension par fil de quartz dans l'électromètre, système extrêmement fragile et fin, nécessite beaucoup de soin, l'aiguille ne devant pas toucher les quadrants. L'électromètre doit être ensuite bien calé, horizontalement, sur une table extrêmement stable, car toute vibration perturbe considérablement le mouvement du spot. Plus tard il sera posé sur une tablette autonome, scellée dans le mur - et, selon certains témoignages, Marie Curie fera même une demande au préfet pour interdire la circulation automobile dans les rues voisines de l'institut du Radium.

Une fois l'aiguille bien immobile et centrée, les 4 quadrants étant à la terre, on la relie à la pile de charge. S'il existe la moindre dissymétrie dans le système, elle subit alors une déviation. Pour la compenser, il est prévu qu'un des couples de quadrants soit mobile dans le 
sens vertical. Il faut donc régler leur hauteur jusqu'à ce que la position de l'aiguille soit indépendante de sa charge. Il faut ensuite installer le dispositif qui envoie le faisceau lumineux sur le miroir, et la règle graduée sur laquelle se réfléchit le spot dont on étudiera le mouvement.

L'installation du quartz piézoélectrique ne nous a pas posé de problème particulier : il faut accrocher la lame de quartz métallisée dans l'appareil, effectuer les branchements, disposer la substance desséchante, et refermer le capot. L'utilisation est décrite plus loin.

Les connexions sont ensuite réalisées. Comme on le voit sur la photographie (Fig. 4), pour les protéger des effets d'influence, on doit utiliser des tubes métalliques, reliés à la terre, au centre desquels sont tendus les fils de connexion, les tubes étant d'assez gros diamètre, et le fil le plus fin possible, afin de réduire la capacité de l'ensemble.

\section{Les chambres d'ionisation}

Comme nous l'avons remarqué par l'étude des carnets, les chambres d'ionisation qu'ont utilisé les Curie pendant l'année 1898 ont été variées, et n'ont pas été conservées pour la plupart : sont mentionnés dans les carnets deux appareils à plateaux, dont un possède un écartement réglable au moyen d'une vis, un plateau à anneau de garde, un appareil cylindrique de plus petite taille, et surtout un "appareil à plateaux sur pieds de cire" qui sera retenu par la suite, et avec lequel l'essentiel des mesures présentées en avril 1898 à l'Académie des Sciences [11] seront réalisées. Ce qui signifie probablement que la question de l'isolation électrique dans la chambre d'ionisation était un des problèmes expérimentaux les plus sérieux (voir plus loin). Lors de la restitution de l'expérience, nous avons utilisé une chambre Beaudoin des années 1920, et une source radioactive scellée d'americium. Cela bien sûr nous éloignait considérablement de la situation de Marie Curie en 1898, qui déposait en couche sur le plateau les produits dont elle étudiait l'activité, avec tous les problèmes que l'on peut imaginer de reproductibilité de l'opération, et de contamination de la chambre par les poussières radioactives (sans parler bien évidemment des conséquences pour la santé des expérimentateurs). C'est en cela également qu'il est impossible de reproduire véritablement les expériences qui ont amené les Curie à isoler le radium.

A travers les carnets, il semble que l'essentiel des difficultés de ces premiers mois - si on se limite, répétons-le, aux questions de la mesure, sans aborder la préparation des sources elles-mêmes, vint de la compréhension de la manière dont la matière radioactive agissait sur la chambre d'ionisation, ainsi que des questions d'isolation. L'écriture de Pierre apparaît dans le carnet durant cette période de mise au point et permet de suivre ses interventions, qui, fin 
janvier - début février 98, semblent surtout concentrées sur des essais de tensions d'alimentation variées pour la polarisation des plaques. Et, plus généralement, la phase de mise au point qui se déroula sur une période de trois mois consista essentiellement à faire varier distance entre les plateaux, et tensions d'alimentation. La variation des courants en fonction de ces paramètres n'obéit en effet pas à une loi simple : lorsque les champs appliqués sont faibles, le courant mesuré est proportionnel à la tension appliquée (comportement ohmique), puis, lorsque le champ est suffisant, on obtient un courant de saturation. Mais ce courant, qui dépend du nombre d'ionisations provoquées par le rayonnement, va d'abord augmenter avec la distance entre les plateaux, puis, si cette distance est trop grande, diminuer à nouveau, à cause de l'absorption par l'air du rayonnement et de la recombinaison des ions produits. Par ailleurs, ce qu'on ne savait pas encore en 1898, le rayonnement est formé de rayons $\alpha, \beta$ et $\gamma$ dont les capacités de pénétration sont bien différentes. Pour compliquer le tout, certains des produits étudiés comme le thorium produisent une émanation gazeuse radioactive qui pouvait progressivement s'accumuler ou s'évacuer suivant la circulation de l'air. On imagine donc la complexité pour retrouver des lois simples, et on comprend qu'il ait fallu le travail de plusieurs équipes différentes pour démêler cet écheveau, dans une période où l'existence même des ions n'était qu'une hypothèse théorique qui ne faisait pas l'unanimité, et où la nature et la cause physique des rayons restait tout à fait mystérieuse.

\section{Les piles}

A la lecture des carnets, la question des piles est une des questions qui a manifestement beaucoup préoccupé le couple, notamment Pierre, durant toute la période de mise au point de l'expérience. Dès le départ, Marie disposait de plusieurs piles formées de 50 éléments au bichromate à électrodes zinc et charbon, qui faisaient probablement partie du banc de mesure développé par Pierre et Jacques Curie, car ce dernier y fait largement référence dans sa thèse : "cette pile était montée dans des petits godets réunis par groupe de 50 et fixés sur des planchettes formant montants, tous les zincs et cuivres étaient fixés à part sur une autre planchette et placés de telle façon qu'on pouvait les immerger facilement au commencement de chaque série d'expériences et les retirer à la fin pour les laver... " [7]. Le bichromate de potassium - produit fortement toxique par ailleurs - réagissait en effet avec le zinc, et il fallait enlever les électrodes dès que les piles n'étaient plus utilisées. Ce qui n'empêcha pas Jacques d'écrire qu'il s'agit de "la plus commode des piles que j'aie employée". Cela permet d'apprécier un des problèmes expérimentaux de l'époque. 
Les éléments au bichromate ont une force électromotrice de $2 \mathrm{~V}$; les $100 \mathrm{~V}$ environ de la pile permettaient donc à la fois la charge de l'aiguille et celle de la chambre. Mais ils n'étaient pas très constants, et, pouvaient varier de $1 \%$ après quelques heures d'utilisation. Pour les étalonnages plus précis, les cahiers Curie mentionnent à la fois des éléments Daniell, plus stables (f.e.m. 1,07 V) et Gouy (f.e.m 1,39V). Mais il était manifestement difficile d'en disposer suffisamment pour remplacer les batteries d'éléments au bichromate.

L'image d'Epinal des conditions précaires dans lesquelles travaillait Marie Curie dans le hangar de la rue Lhomond n'est pas usurpée. Les cahiers mentionnent à plusieurs reprises pendant le mois de février 1898 des températures de l'ordre de $6^{\circ} \mathrm{C}$. Quelques semaines plus tard, elles dépassaient $16^{\circ} \mathrm{C}$. Cela n'avait pas trop d'influence sur les phénomènes étudiés, la radioactivité ne dépendant pas de la température, mais cela en avait sur la force électromotrice des piles... Après de multiples essais, à partir du 16 février, toutes les mesures seront faites avec des champs suffisamment élevés (une pile, c'est-à-dire $100 \mathrm{~V}$, pour $3 \mathrm{~cm}$ ) pour avoir le courant de saturation dans la chambre. Cela permettait de minimiser l'influence des piles.

Au moment de la réplication de l'expérience, nous n'avions pas pris conscience de l'importance de ce problème, et nous avons alimenté pile et chambre avec une source de tension moderne.

\section{Faire appel à la mémoire vivante ; la manipulation du poids}

Nous avons pu interroger plusieurs personnes qui avaient utilisé le montage à l'Institut du Radium dans les années 1950 : lors de la reconstitution de l'expérience, Maurice Duquesne notamment se rappelait des travaux pratiques qu'il encadrait sous la houlette de R.Grégoire, vers 1956. Le banc de mesure a continué à être utilisé jusqu'en 1964, à l'époque où il a été abandonné, étant démodé et surtout trop délicat pour les étudiants...

D'après la notice des instruments Beaudoin de 1927 [12], étayée par ces témoignages, la meilleure manière d'effectuer la mesure est la suivante : un poids étalon étant posé de manière bien stable sur le plateau, l'expérimentateur ouvre l'interrupteur, ce qui permet la déviation du spot sous l'influence du courant d'ionisation de la chambre. Il déclenche le chronomètre à une position du spot qu'il repère sur la règle, puis, le coude posé sur la table, il allège le poids le plus régulièrement possible de manière à maintenir le spot au zéro. Au moment où le poids quitte le plateau, le spot reprend sa déviation. L'expérimentateur doit alors arrêter le chronomètre lorsque le spot passe à nouveau à la position repérée.

Tous les témoignages que nous avons recueillis s'accordaient sur la dextérité nécessaire à ces mesures. Il ne faut cependant peut-être pas exagérer la difficulté concernant l'utilisation du 
quartz et de l'électromètre eux-mêmes. Nous avons pu nous familiariser sans trop de difficultés avec son mode opératoire, et les carnets ne font pas état de difficultés de Marie Curie de ce côté là.

\section{La question de l'isolation électrique}

Une question autrement plus délicate, qui se retrouve tout au long des carnets semble être en revanche celle de l'isolation. Le premier essai consigné par Marie sur un carnet, le 16 décembre 1897, se termine par la phrase "l'électromètre fuit". L'intervention de Pierre fut en grande partie consacrée à ces problèmes d'isolation. Le 4 février, une phrase du carnet permet de chiffrer la fuite de la chambre d'ionisation, à environ 1 pA, c'est à dire non négligeable par rapport aux courants mesurés. Suit la phrase "on perfectionne le dessèchement".

Il faut prendre des précautions pour le quartz, dont la lame doit avoir des bords propres, et qui doit être conditionné pendant plusieurs heures avec un produit desséchant [12]. L'électromètre également doit être préparé de cette manière. Mais il semble que c'est la chambre qui a posé le plus de problèmes de courants de fuite. Du fait de l'utilisation de la méthode de zéro, c'est le seul appareil qui est soumis à une différence de potentiel lors de la mesure, de l'ordre de 100 volts, et de plus, les substances radioactives finissent par se disséminer dans tout le laboratoire, de sorte que l'air lui-même devient conducteur. Pierre Curie écrit en 1900 : "dans le laboratoire où nous travaillons, le mal est arrivé à l'état aigu et nous ne pouvons plus avoir un appareil bien isolé. " [13] ; pour les liaisons entre appareils, on ne peut plus faire confiance à des cylindres remplis d'air, et il faut utiliser des diélectriques solides. Par ailleurs, il faut utiliser des électromètres rigoureusement clos.

\section{Analyse des mesures retranscrites dans le carnet ; sensibilité du système}

On présente ici (Fig. 5) un extrait assez représentatif des séries de mesures consignées par Marie Curie sur son cahier, qui feront l'objet de sa première communication à l'Académie des Sciences en avril 1898. La mesure de déviation lors de l'application d'un poids de 50 grammes sert probablement à vérifier le maintien depuis la veille de la bonne isolation du système : on note qu'il y a une déviation au moment de la pose du poids, puis le spot se stabilise à une valeur un peu inférieure, la différence étant probablement due à la polarisation non instantanée des diélectriques. Le spot doit alors ne revenir que très lentement au zéro.

Une ligne de mesure se présente ensuite de la manière suivante : la colonne de gauche donne le poids utilisé, soit ici 500 grammes, qui est le poids le plus élevé utilisé, la pechblende étant particulièrement active. La deuxième colonne donne le temps mesuré avec la 
technique explicitée au paragraphe précédent, et la troisième donne la valeur du "courant", mesuré en gramme par seconde, quotient des deux premières valeurs. Aucune conversion de ce quotient en ampères n'apparaît dans les carnets eux-mêmes, mais une confrontation méticuleuse avec la publication d'avril 1898 (donnée en pA) nous a permis d'établir que le coefficient du quartz est de l'ordre de 2,1 pC par gramme. Les courants mesurés pour la pechblende grise correspondent donc à $13 \mathrm{pA}$.

Une des premières mesures consignées dans le carnet permet de connaître la sensibilité de l'électromètre : l'application d'un élément Leclanché (f.e.m. 1,5 V) donne une déviation de 40 cm, ce qui donne $37,5 \mathrm{mV} / \mathrm{cm}$. La confrontation avec les données fournies par Ledeboer [3] montre qu'il est possible que l'électromètre utilisé ait été le modèle Curie apériodique construit par la SCPC, représenté en Fig.3. Par ailleurs, la connaissance par les carnets (Fig. 5) de la déviation $(21 \mathrm{~cm}$ ) lors de l'application d'un poids de $50 \mathrm{~g}$, (soit $105 \mathrm{pC}$ ) permet de reconstituer la capacité de l'ensemble, qui est $\mathrm{C}=\mathrm{Q} / \mathrm{V}=133 \mathrm{pF}$.

On constate donc que tout le dispositif est très sensible. D'ailleurs, à plusieurs reprises, on voit dans les carnets que Marie Curie est gênée par la présence de forces électromotrices spontanées, probablement des différences de potentiel de contact à différents endroits du circuit. Mais elle a à sa disposition un système de mesure dont la précision et la fiabilité restera probablement sans équivalent pendant plusieurs décennies.

\section{Conclusions}

Avec le développement des recherches en radioactivité, bien des techniques de mesures vont se répandre, dont aucune n'avait la précision des méthodes électrométriques: elles répondaient à d'autres exigences, essentiellement pratiques. De multiples électroscopes seront ainsi développés (y compris par Pierre Curie) pour satisfaire aux besoins du terrain, pour la mesure de la radioactivité des minerais ou celle des engrais radioactifs développés dans l'agriculture dans les années où on attribuait à la radioactivité de grandes vertus. Des méthodes fondées sur des principes tout à fait différents vont également apparaître, comme la méthode des scintillations ou encore le compteur Geiger en 1908, répondant au besoin de procéder au comptage des particules $\alpha$, mais elles resteront longtemps peu fiables et peu reproductibles.

Les méthodes électrométriques resteront donc largement dominantes dans les laboratoires de recherche, et considérées comme des méthodes de précision. Mais il est curieux de 
constater que le dispositif de mesures décrit dans cet article, associant le quartz à l'électromètre piézoélectrique, restera une spécificité française, voire même une spécificité "Curie", alors qu'il avait permis des découvertes importantes. La méthode de mesure des Curie aura sans doute vu sa diffusion limitée par sa fragilité et sa relative complexité, mais plus encore sans doute, par les traditions locales des quelques laboratoires de référence dans le domaine : Lord Kelvin, à qui Pierre Curie avait envoyé un quartz piézoélectrique dans les années 80, avait été déconcerté par son utilisation et l'avait laissé de côté. Effet du climat de Glasgow, ou manque de motivation ? Quant à Ernest Rutherford et aux chercheurs du Cavendish à Cambridge, il semblerait qu'ils n'aient pas eu besoin d'une telle précision dans les mesures, et ils ont continué à utiliser des mesures de déviation.

Cela n'a pas empêché la méthode de mesure des Curie de rester longtemps une référence pour les chercheurs en radioactivité du monde entier. Lorsqu'il s'est agi de mettre en place une métrologie internationale de la radioactivité, c'est sur la base de cette méthode qu'elle a été élaborée; c'est d'ailleurs Marie Curie qui a été chargée de la réalisation de l'étalon primaire du radium en 1910. Dans les années 1920, cette technique passa progressivement au second plan, car le champ de la radioactivité connut des mutations importantes. Alors que les travaux initiaux étaient dominés par la définition des caractéristiques des radioéléments et de leurs rayonnements, petit à petit la question de la structure des atomes et de la nature des particules qui les composent prit une plus grande importance. Sa résolution suscita de nouvelles techniques expérimentales, comme le compteur de Geiger-Müller en 1928, et la chambre à brouillard. C'est une autre histoire qui commençait, celle de la physique nucléaire.

\section{Références}

[1] M. Curie, P. Curie, Carnet d'analyse bibliographique, Bibliothèque nationale de France, Fonds Curie, n.a.f 18382.

[2] Lord Kelvin, J. C. Beattie, S. Smoluchowski, Conductance produced in Gases by Röntgen rays, ultra-violet light and by uranium, and some consequences thereof, Philosophical Magazine, 43 (1897), p. 418-439.

[3] P. H. Ledeboer, Nouveaux électromètres à quadrants apériodiques, La Lumière électrique, 22 (1886), reproduit dans Oeuvres de P.Curie, Gauthier-Villard 1908, p564.

[4] E. Mascart, Traité d'électricité statique, Masson 1876. 
[5] Lord Kelvin, Report on electrometers and Electrostatic measurements, 1867 in : Reprint of papers on electrostatics and magnetism, Macmillan, 1872.

[6] P. Curie, J. Curie, Les cristaux hémièdres à faces inclinées, comme sources constantes d'électricité, CR. Acad. Sci. , 93 (1881), p. 204.

[7] J. Curie, Recherches sur le pouvoir inducteur spécifique et sur la conductibilité des corps cristallisés, (Thèse Fac. Sci. Paris, Imprimerie des Lumières électriques, 1888).

[8] P. Curie, Equations réduites pour le calcul des mouvements amortis, La Lumière électrique, 41 (1891), in : Oeuvres de P.Curie, Gauthier-Villard 1908, p158.

[9] M. Curie, P. Curie, Carnets de la découverte, Bibliothèque nationale de France, Fonds Curie, n.a.f 18379, Microfilms nº4083 à 4086.

[10] S. Boudia, Marie Curie et son laboratoire : science et industrie de la radioactivité en France, Editions des archives contemporaines, Paris, 2001.

[11] M. Curie, Rayons émis par les composés de l'uranium et du thorium, CR.Acad.Sci., 126 (1898), p. 1101-1103.

[12] C. Beaudoin, Appareils pour mesure de radioactivité, notice du constructeur Ch.Beaudoin, 1927

[13] M. Curie, P. Curie, Les nouvelles substances radioactives et les rayons qu'elles émettent, Rapport au Congrès Int. de Physique, 1900, reproduit dans Oeuvres de P.Curie, GauthierVillard, 1908 p407-408. 


\section{Liste des figures}

Fig. 6 : Aiguille et quadrants de l'électromètre

Fig. 7 : Electromètre à quadrants Kelvin

Fig.8 : Quartz piézoélectrique, et électromètre apériodique Curie

Fig. 9 : Le célèbre hangar de la découverte, avec le banc de mesure

Fig. 10 : Schéma du montage et extrait d'une page du cahier de mesures de Marie Curie 